\title{
EDITORIAL
}

\section{WHITHER OPHTHALMOLOGY?}

The College of Ophthalmology is now five years old and even during its short life span there has been an enormous change in the breadth of knowledge of the function of the eye, its central connections, the perception of vision and the changes which result from malfunction of the various parts. These advances have come not only from basic scientific research in ophthalmology, visual science and technology but also from advances in the fields of genetics, molecular biology, immunology, connective tissue and neurology.

The changes are happening so fast that it is difficult to decide where each new discovery fits into the function and dysfunction of the eye and its connections. It is the major purpose of Eye to present as much of this information as possible to its readers in a digestible form in order that they can relate it to their practice and, it is hoped, use it to improve the management of the patients under their care. Unfortunately, this is becoming more and more difficult to achieve. Whilst support can easily be obtained to increase the speed at which straightforward cases are fed through the system there is, in spite of protestations to the contrary, less and less support being given to those who have to deal with the more complex, time-consuming and more severely blinding diseases which are the ones that are likely to be the beneficiaries of continuing ophthalmic research.

If this trend continues and the only thing that comes to matter is the 'cost/benefit ratio' then ophthalmology will become an isolated speciality with no right to represent itself in the academic community. Short-term benefits and obsession with improving surgical techniques must never obscure the long-term gain of continual research and development, however great the temptation.

P. G. WATSON 